\title{
Oncogenic signaling upstream of mTORC1 drives lipogenesis and proliferation through SREBP
}

\author{
Stéphane Ricoult ${ }^{1 *}$, Jessica Yecies ${ }^{2}$, Brendan Manning $^{1}$ \\ From Metabolism, Diet and Disease 2014: Cancer and metabolism \\ Washington DC, USA. 28-30 May 2014
}

\section{Background}

The mechanistic target of rapamycin (mTOR) is a central regulator of cell growth and proliferation, and its aberrant activation is frequent in cancer [1]. We have previously shown that sterol regulatory element binding protein (SREBP) is a major transcriptional effector of mTOR complex 1 (mTORC1) signaling [2]. SREBP is a transcription factor that stimulates the expression of genes involved in the de novo synthesis of lipids [3]. Since mTORC1 is commonly activated in cancer through upstream oncogenic signaling pathways and enhanced lipogenesis is a metabolic hallmark of tumor cells, we hypothesize that the mTORC1-SREBP pathway is important for tumor metabolism and growth.

\section{Materials and methods}

We used a panel of breast cancer cell lines with various mutations resulting in constitutive mTORC1 activation, as well as MCF10a cells transformed with oncogenic KRAS or PIK3CA to explore the role of SREBP in mTORC1-driven cancers. We used chemical inhibitors and siRNAs to assess the dependence of our breast cancer systems on the mTORC1-SREBP pathway for de novo lipogenesis, proliferation, growth, and cell survival.

\section{Results}

Using both the panel of breast cancer cell lines and the isogenic MCF10a cell lines, we have revealed an essential role for SREBP in driving lipogenesis and proliferation in tumor cells. Our results indicate that SREBP and its lipogenic targets are activated in cell lines with oncogeneinduced mTORC1 signaling, and this activation was ablated by mTOR inhibitors. mTORC1 signaling promotes de novo lipogenesis in an SREBP-dependent manner in cancer cells. In addition, we found that the mTORC1stimulated activation of SREBP2 promotes cell growth, proliferation, and survival of breast cancer cells. We are in the process of identifying the essential functions of SREBP2 in breast cancer and assessing the dependence of breast cancer cells on SREBP2 in vivo. These findings emphasize the therapeutic potential of targeting lipid metabolism in cancers, particularly those with activated mTORC1 signaling.

\section{Authors' details}

${ }^{1}$ Genetics and Complex Diseases, Harvard School of Public Health, Boston, MA, USA. ${ }^{2}$ Genentech, South San Francisco, CA, USA.

Published: 28 May 2014

References

1. Menon S, Manning BD: Common corruption of the mTOR signaling network in human tumors. Oncogene 2008, 27:S43-S51.

2. Düvel $K$, Yecies JL, Menon S, Raman P, Lipovsky Al, Souza AL, Triantafellow E, Ma Q, Gorski R, Cleaver S, Vander Heiden MG, Mackeigan JP, Finan PM, Clish CB, Murphy LO, Manning BD: Activation of a metabolic gene regulatory network downstream of mTOR complex 1. Mol Cell 2010, 39:171-183.

3. Ricoult SJH, Manning BD: The multifaceted role of mTORC1 in the control of lipid metabolism. EMBO Rep 2013, 14:242-251.

doi:10.1186/2049-3002-2-S1-P62

Cite this article as: Ricoult et al:: Oncogenic signaling upstream of mTORC1 drives lipogenesis and proliferation through SREBP. Cancer \& Metabolism 2014 2(Suppl 1):P62. 